\title{
Level of Depression in Temporomandibular Disorder Patients
}

\author{
Tooba Saeed ${ }^{1}$ \\ Nabeela Riaz $^{2}$
}

\author{
FCPS \\ FCPS, MCPS
}

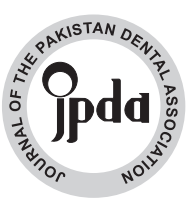

OBJECTIVE: Temporomandibular disorders (TMDs) are common musculoskeletal pain conditions characterized by pain in the temporomandibular joint (TMJ) and/or the masticatory muscles. Due to the complexity of the masticatory system, TMD symptoms may be caused by different physiological and/or psychosocial factors. The purpose of this study is to determine the level of depression in patients with TMD. These findings can guide the diagnosis, prevention, and treatment of TMDs.

METHODOLOGY: In a prospective study, participants were screened and underwent a baseline physical examination of the head and neck, conducted according to the protocol of Research Diagnostic Criteria Axis I for Temporomandibular Disorders(RDC/TMD)(1), in the department of oral and maxillofacial surgery, King Edward medical university, Mayo hospital Lahore from Aug, 2017 to Dec, 2017. 150 patients completed psychological questionnaire Beck's Depression Inventory, to assess the level of depression in patients with TMDs.

RESULTS: Among patients, moderate depression was more frequent i.e., 30.7\%. Severe depression was $22.0 \%$ while extreme depression was $16 \% .10 \%$ of patients were with borderline clinical depression.

CONCLUSION: A substantial number of TMD patients had moderate to extreme level of depression that could be contributory factor in their disease process, but further research on more select groups of TMD patients is needed. KEY WORDS: Temporomandibular disorders, etiology, depression, Psychological

HOW TO CITE: Saeed T, Riaz N. Level of depression in temporomandibular disorder patients. J Pak Dent Assoc 2018;27(3):100-05.

DOI: https://doi.org/10.25301/JPDA.273.100

Received: 23 January 2017, Accepted: 05 March 2018

\section{INTRODUCTION}

$\mathrm{T}$ emporomandibular disorders (TMDs) are musculoskeletal ${ }^{1}$ pain conditions characterized by pain in the temporomandibular joint (TMJ) and/or the masticatory muscles. ${ }^{2}$ The prevalence of TMD symptoms among the general population is around $40 \%{ }^{3}$ Epidemiological studies show that $50-75 \%$ of the persons in a certain population have at least one sign of TMDs during their life, while $20-25 \%$ of the persons suffer from significant symptoms related to the TMDs. ${ }^{4}$ The most important symptom is pain, followed by restricted mandibular movements, which can cause difficulty in eating or speaking; noises from the temporomandibular joints during jaw movement are also recorded. The etiology and pathogenesis of this condition is poorly understood, therefore treatment of temporomandibular joint diseases is sometimes difficult. Understanding the etiology of temporomandibular joint disorders is extremely important in identifying and avoiding

1. Senior Registrar, Department of Oral and Maxillofacial Surgery, The University of Lahore.

2. Associate professor, HOD, Department of Oral and Maxillofacial Surgery, King Edward Medical University/Mayo Hospital Lahore.

Corresponding author: "Dr. Tooba Saeed"<drtoobasaeed@yahoo.com > potential pathologic factors. ${ }^{5}$ Due to the complexity of the masticatory system, TMD symptoms may be caused by different physiological and/or psychosocial factors, such as malocclusion and occlusal interferences, alterations in the masticatory muscles, direct trauma to the jaw or TMJ, microtrauma caused by continuous parafunctional habits or alterations secondary to stress. ${ }^{3}$

The role of stress and personality in the etiology of the temporomandibular pain dysfunction syndrome has undergone extensive scrutiny. Psychological studies have shown that patients with TMD have similar psychological profiles and psychological dysfunction as other chronic musculoskeletal pain disorders, such as tension type headache and back or arthritic pain. There is considerable evidence that psychological and psychosocial factors are of great importance in the understanding of TMD, but there is less evidence that these factors are etiologic. ${ }^{5}$

Laskin $^{6}$ was the first to suggest that the main factor responsible for TMD is the emotional instead of the physical aspect. During the last decade, numerous investigations have been devoted to understand the relationship between psychological stress and TMD. Patients suffering from this condition report that their symptoms increase during stressful 
situations . De Leeuw et $\mathrm{al}^{7}$ consider that muscle dysfunction and accompanying pain are very often the result of stress induced muscular hyperactivity. Stress-induced muscular dysfunction may induce secondary changes in the TMJ. Raised elevator tonus leads to increased intraarticular pressure in TMJ and alteration in the normal biomechanics, resulting in microtraumatic damage to the joint capsules and disk attachment. However, the studies that investigate psychological factors present mixed results. Some investigators related electromyographic changes in masticatory muscle baseline values between patients with TMD and control individuals, while others did not find significant differences in electromyographic activity baseline values between patients and controls. These inconsistencies may be probably due to different methodologies used. ${ }^{8}$ Nowadays the association between depression and stress and different physical symptoms of TMD is widely acknowledged. ${ }^{5}$ The purpose of this study is to determine the level of depression in patients with TMD. These findings can guide the diagnosis, prevention, and treatment of TMDs.

\section{METHODOLOGY}

We undertook a prospective study, on patients who presented with Temporomandibular disorders (TMDs), in the department of oral and maxillofacial surgery, King Edward medical university, Mayo hospital Lahore from Aug, 2017 to Dec, 2017. Prior to enrollment in the study, participants were screened and underwent a baseline physical examination of the head and neck, conducted according to the research diagnostic criteria (RDC/TMD) for TMD. ${ }^{1} 150$ patients completed psychological questionnaire with their informed consents. Questionnaire selected was Beck's Depression Inventory, to assess the level of depression in patients with TMDs. Variables were Patients age, gender and level of depression. Data was entered in SPSS. We also categorized patients in 15-30 year age group and the 31-50 year age group. Chi-square test was used to determine statistically significant difference in level of depression among age groups and male female patients $(p<0.05)$.

\section{RESULTS}

Mean age of patients was 31years, with minimum age 17 years and maximum 50 years. Of them, in age group 15-30 years, there were 91 (60.7\%) patients. While, 59(39.3\%) patients were belonged to 31-50 years age group( Fig 1). Out of total 150 patients, 93 (62\%) were females and 57(38\%) were males (Fig 2). Among females $69.9 \%$ were from 15-30 years age group, while $45.6 \%$ of males were belonged

\begin{tabular}{|l|c|c|}
\hline & Frequency & Percent \\
\hline $\begin{array}{l}\text { These ups and downs are } \\
\text { considered normal }\end{array}$ & 25 & 16.7 \\
Mild mood disturbance & 7 & 4.7 \\
Borderline clinical & 15 & 10.0 \\
depression & 46 & 30.7 \\
Moderate depression & 33 & 22.0 \\
Severe depression & 24 & 16.0 \\
Extreme depression & 150 & 100.0 \\
Total & & \\
\hline
\end{tabular}

Table 1: Patient Level of Depression

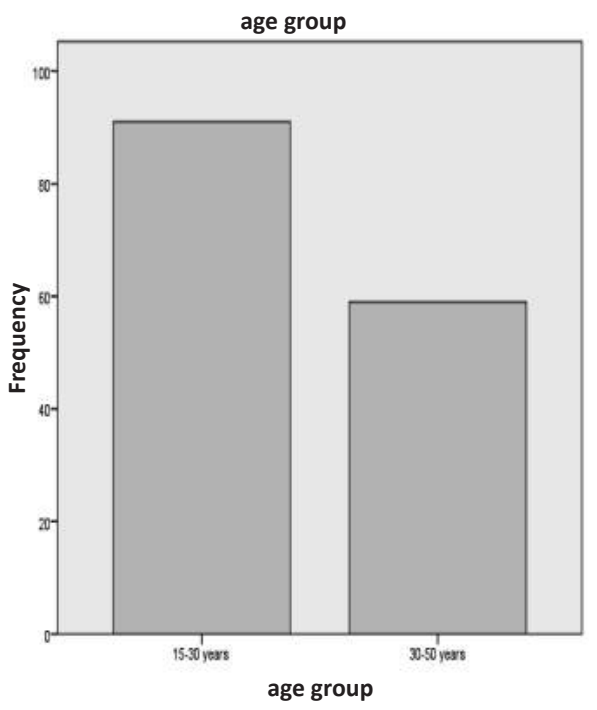

Fig 1: Frequency of age groups in TMD patients

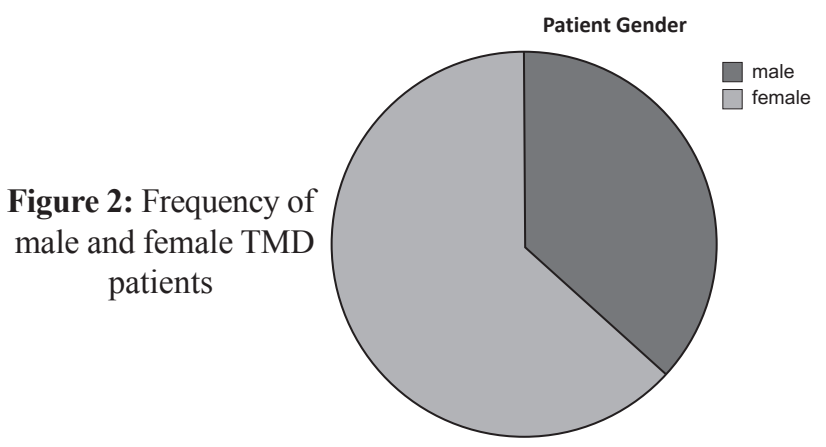

Fig 2: Frequency of male and female TMD patients

to15-30 years age group. But the difference was not significant statistically. Among patients, moderate depression was more frequent i.e., $30.7 \%$. Severe depression was $22.0 \%$ while extreme depression was $16 \% .10 \%$ of patients were with borderline clinical depression (Fig 3). Moderate and severe depressions were significant in female patients. In females 


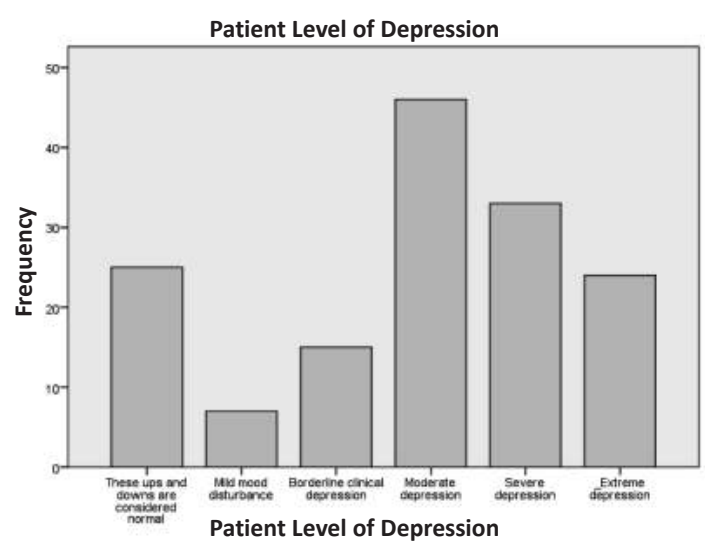

Fig 3: Frequency of Patient Level of Depression

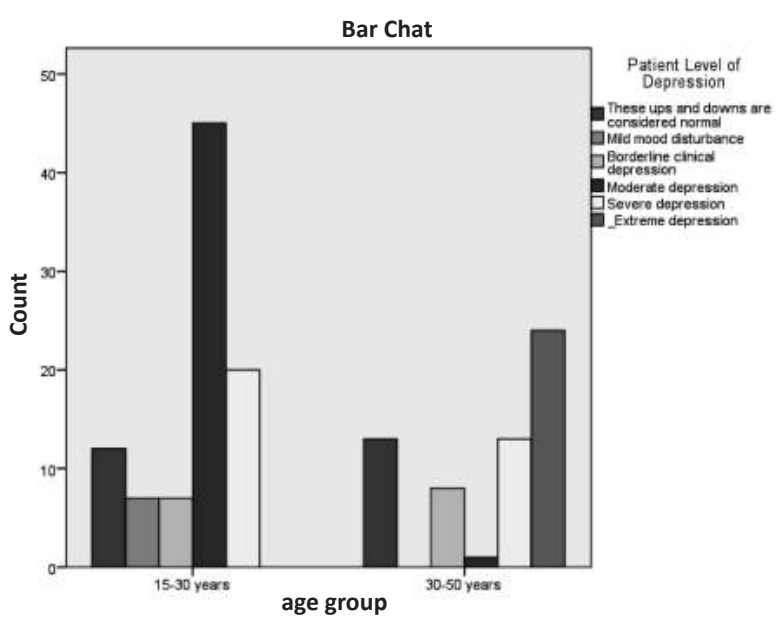

Fig 4: Level of depression in Age groups

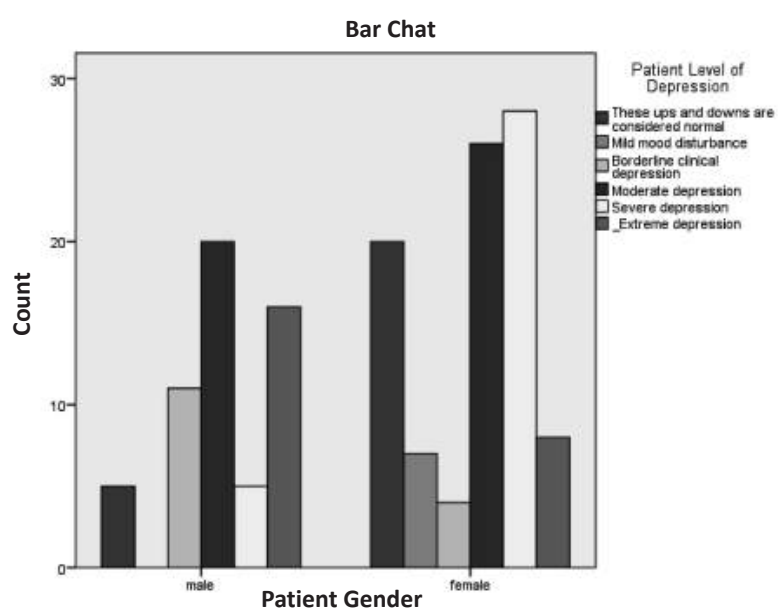

Fig 5: Level of depression among male and female patients

moderate depression was $28 \%$, while severe depression was $30.1 \%$. Extreme depression in females was only $8.6 \%$. On contrary, extreme depression was more significant in males,
$28.1 \%$. In male patients, moderate depression was $35.1 \%$ and severe depression was only $8.8 \%$. In $21.5 \%$ of females, there were normal ups and downs in mood. While $8.8 \%$ of males had normal ups and downs in mood (Fig 5). Similarly, mild mood disturbance in females was relatively more pronounced, $7.5 \%$. Border line clinical depression was $19.3 \%$ in males and $4.3 \%$ in female patients. Level of depression is significantly different in age groups and male female patients i.e, $p=0.01$ and $p=0.04$ respectively.

\section{DISCUSSION}

Over the last 75 years, a variety of etiologic factors has been suggested as the cause of pain and dysfunction in the temporomandibular system. The earliest and still-popular etiologic theory proposed that temporomandibular disorders are induced by abnormal structure, usually described as a malocclusion of the teeth or jaws. The fact that this theory was based on mechanical concepts, ignored biologic diversity, and had limited factual experimental evidence to support it as well as extensive evidence in opposition did not seem to matter to its proponents. In the late 1960's and early 1970's, the structural occlusal model for TM Disorders was challenged and has yielded ground to a more multifactorial model of TMD causation. Other etiologic factors for TM disorders-such as anatomical susceptibility of TM tissues to trauma, polyarthritic diseases, joint laxity, repetitive parafunctional behaviors, and stress-related muscle dysfunction--were recognized. ${ }^{9}$ Importance of the psychological factors for development of the TMD grows every day and is potentiated by the inability of proving any other valid etiological factors. According to Green ${ }^{10}$ unresolved psychological issues, such as depression and anxiety, can cause tension which inevitably leads to bruxism and parafunctions, which in turn cause TMD. Psychological factors as etiological factors in development of the TMD cannot be ignored, since they play a great role in many painful conditions in the organism, including the TMD. Kinney et $\mathrm{al}^{11}$ in their research noted that the psychological disorders are the key factors in the development of TMD. The most recent investigations on TMD focus on the relationship between the physical and psychological factors. A number of published papers proved the relationship between the TMD and anxiety, depression and stress, but they failed to reveal the cause of this relationship. ${ }^{12}$

Most studies on TMDs had been carried out in developed countries, and little is known about TMDs in developing countries such as Pakistan for development of standardized clinical measures, have been suggested to improve reliability, potentially and validity of clinical examinations. The Research Diagnostic Criteria for TMD (RDC/TMD) was established 
to allow standardization and replication of the most common forms of research relating muscle and joint. ${ }^{13}$ Since $\mathrm{RDC} / \mathrm{TMD}$ is an internationally recognized and widely adopted tool for TMD research, its methodology was used in this study. ${ }^{1,2}$

These data essentially support and extend prior studies, which have shown that TMD patient samples report relatively more psychologic and somatic symptoms. Most previous studies, however, have used ill-defined control groups. ${ }^{14}$ TMDs have been reported to primarily affect young and middle-aged adults, and they are approximately twice more common in women than in men in the general population. ${ }^{15}$ These findings are in accordance with our study. Mean age group of TMDs patient was 31years, females were $62 \%$ while males were $38 \%$. Diracoglu (2016) revealed in his study that, among the patients with TMD, the groups who were considered to have anxiety and depression were female patients, patients with deficient social support system, and patients with myofacial pain alone or patients with myofacial pain accompanying an existing TMJ disorder. ${ }^{16}$ Both the women and men TMD patients in the present study reported significantly more and/or more frequent somatic, psychologic, and behavioral symptoms on the Beck's Depression Inventory, on average. Thus, there is some justification for concluding that the results of prior studies using largely female TMD patient samples might be generalized to male TMD patient samples.

Individuals with TMD are more anxious and depressive than asymptomatic ones, and TMD symptoms have their onset and are exacerbated in periods of psychological stress. In addition, pain on the temporomandibular joints has been associated with general anxiety. ${ }^{17}$ TMD pain is the commonest symptom that compels patients to seek therapy. The prevalence of the signs and symptoms of TMD has been reported to vary from 6 to $93 \%$, while only 3.6 to $7 \%$ of the general populations have been estimated to be in need of treatment. These wide ranges of prevalence may be probably due to different criteria and methodologies used..$^{8}$ In TMDs, treatment is directed to the elimination of the pain and/or dysfunction such as limitation. Medicines such as analgesics and anti-inflammatory drugs, psychological consultation, TMJ imaging is necessary when the symptoms persist or worsen during the treatment. For cases with only clicking and/or deviation, TMJ imaging is not necessary. Different intraoral appliances and splints, and arthrocentesis (hydraulic distension) have been used for elimination of the etiologic factor(s) of the complaints. ${ }^{15}$

Bertoli (2016) reported elevated levels of suicidal ideation, depression, and anxiety in a chronic TMD population, especially in those with chronic muscle pain, compared to the general population. He emphasized the need for screening for suicidality and other comorbidities in TMD patients suffering from chronic pain. ${ }^{18}$ Brandini et al in his exploratory study has provided data suggesting that psychological factors, manifesting in depression and stress, play a role in influencing the association between pain and motor activity. In his study, there were significant $(\mathrm{P}<.05)$ positive correlations between depression and jaw amplitude and stress and jaw velocity for standardized but not free chewing. ${ }^{19}$ Some recent reports $^{2,20,21}$ also prove the multicausal etiology of the TMD, although anxiety and depression may also arise from some organic pathological condition. It is possible that in some patients the predisposing role is played by psychological factors coupled with a decreased tolerance to pain threshold and reduced tolerance to stress. Lajnert V(2010), recommended introduction of psychological therapy for the chronic painful conditions. Besides the traditional psychotropic drugs (trycyclic antidepressants), introduction of psychological intervention, stress management and change of habits as a part of the integral treatment of TMD patients with depression, can be effective in reduction of painful conditions and tension in various types of patients suffering from TMD. ${ }^{4}$

Velly AM,(2011) found a moderately positive correlation between depression and catastrophizing, which will in turn contribute to an increase in pain intensity, disability, and the onset and progression of clinically significant pain. These findings were not surprising since catastrophizing is an element of the distorted cognitive processes associated with clinical depression. It should also be noted that symptoms of depression are reported in a number of studies with TMD pain patients. They found that catastrophizing and depression both constitute factors that will complicate management of TMD and thus, are needed to be considered in both evaluation and management of TMD. Simple cases without or with minimal comorbid conditions involvement can be managed by a single clinician with single treatments. Nevertheless, it is critical to match the level of complexity of the management program with the comorbid conditions reported by the patient. Failure to identify and to address the entire scope of the problem may lead to no improvement in pain or function, and further perpetuation of the problem. Once identified, complex TMD patients, particularly those with comorbid conditions, can best be managed within an interdisciplinary pain clinic setting. ${ }^{22}$ Fernandes Azevedo $\mathrm{AB}$, study results corroborates a previous paper ${ }^{23}$, which stated that no significant association could be found between anxiety and disorders in general. However, many authors ${ }^{24-26}$ found a significant association between anxiety and TMD. This finding may be explained by methodological differences, since some studies comprised a sample of nonpatients, differing from the papers that measured anxiety 
levels in subjects seeking treatment. ${ }^{17}$

Some limitations in the present study need to be pointed out. First, standard questionnaires were used in the present study. However, inaccuracies may always occur when gathering data with questionnaires. The reporting of symptoms may depend on several factors, e.g., subjects' motivation; therefore, comparing data of different studies may include bias due to cultural or societal differences. Sometimes individual with different kinds of stress may be more likely to perceive, over-react and complain about their sensations during reporting of symptoms. Also we did not categorized patients according to etiology of TMD, like mayofacial pain vs nonmayofacial pain groups and severity of TMD, like like acute vs chronic TMD patients.

\section{CONCLUSION}

TMD patients experienced different Level of depression from no depression to extreme depression. Moderate, severe and extreme depressions were remarkable among TMD patients. But further research on more selected groups of TMD patients is needed, including controlled studies of pharmacological and/or psychotherapeutic intervention, to clarify the role of depression in this condition.

\section{CONFLICT OF INTEREST}

There is no conflict of interest and funding.

\section{REFERENCES}

1. Dworkin SF, LeResche L. Research diagnostic criteria for temporomandibular disorders: review, criteria, examinations and specifications, critique. J Craniomandib Disord. 1992;6(4):301-55.

2. Akhter R, Hassan NM, Aida J, Kanehira T, Zaman KU, Morita M. Association between experience of stressful life events and musclerelated temporomandibular disorders in patients seeking free treatment in a dental hospital. Eur J Med Res. 2007;12(11):535-40.

3. Calixtre LB, Gruninger BL, Chaves TC, Oliveira AB. Is there an association between anxiety/depression and temporomandibular disorders in college students? Journal of applied oral science : revista FOB. 2014;22(1):15-21.

https://doi.org/10.1590/1678-775720130054

4. Lajnert V, Franciskovic T, Grzic R, Kovacevic Pavicic D, Bakarbic D, Bukovic D, et al. Depression, somatization and anxiety in female patients with temporomandibular disorders (TMD). Coll Antropol. 2010;34(4):1415-9.

5. Chisnoiu AM, Picos AM, Popa S, Chisnoiu PD, Lascu L, Picos A, et al. Factors involved in the etiology of temporomandibular disordersa literature review. Clujul medical (1957). 2015;88(4):473-8. https://doi.org/10.15386/cjmed-485

6. Laskin DM. Etiology of the pain-dysfunction syndrome. J Am Dent Assoc. 1969;79(1):147-53.

https://doi.org/10.14219/jada.archive.1969.0234

7. de Leeuw JR, Steenks MH, Ros WJ, Lobbezoo-Scholte AM, Bosman F, Winnubst JA. Multidimensional evaluation of craniomandibular dysfunction. I: Symptoms and correlates. J Oral Rehabil. 1994;21(5):501-14.

https://doi.org/10.1111/j.1365-2842.1994.tb01165.x

8. Gameiro GH, da Silva Andrade A, Nouer DF, Ferraz de Arruda Veiga MC. How may stressful experiences contribute to the development of temporomandibular disorders? Clin Oral Investig. 2006;10(4):261-8. https://doi.org/10.1007/s00784-006-0064-1

9. Clark GT. Etiologic theory and the prevention of temporomandibular disorders. Adv Dent Res. 1991;5:60-6.

https://doi.org/10.1177/08959374910050010901

10. Fraley SE. Efficacy of an Early Biopsychosocial Intervention for Patients with Acute Temporomandibular Disorder-Related Pain: a Sixto Ten-Year Follow Up Study 2011.

11. Kinney RK, Gatchel RJ, Ellis E, Holt C. Major psychological disorders in chronic TMD patients: implications for successful management. The Journal of the American Dental Association. 1992;123(10):49-54.

https://doi.org/10.14219/jada.archive.1992.0256

12. Manfredini D, Landi N, Bandettini Di Poggio A, Dell'Osso L, Bosco M. A critical review on the importance of psychological factors in temporomandibular disorders. Minerva Stomatol. 2003;52(6):321$6,7-30$.

13. Yap AU, Tan KB, Prosthodont C, Chua EK, Tan HH. Depression and somatization in patients with temporomandibular disorders. The Journal of prosthetic dentistry. 2002;88(5):479-84.

https://doi.org/10.1067/mpr.2002.129375

14. Beaton RD, Egan KJ, Nakagawa-Kogan H, Morrison KN. Selfreported symptoms of stress with temporomandibular disorders: comparisons to healthy men and women. J Prosthet Dent. 1991;65(2):289-93. https://doi.org/10.1016/0022-3913(91)90177-X

15. Sener S, Akgunlu F. Sociodemographic comparison in patients with subjective and objective clinical findings of temporomandibular dysfunctions. European journal of dentistry. 2011;5(4):380.

16. Diracoglu D, Yildirim NK, Saral I, Ozkan M, Karan A, Ozkan S, et al. Temporomandibular dysfunction and risk factors for anxiety and depression. J Back Musculoskelet Rehabil. 2016;29(3):487-91. https://doi.org/10.3233/BMR-150644

17. Fernandes Azevedo AB, Câmara-Souza MB, Dantas IdS, de Resende CMBM, Barbosa GAS. Relationship between anxiety and temporomandibular disorders in dental students. CRANIOß. 2017:1-4. 
https://doi.org/10.1080/08869634.2017.1361053

18. Bertoli E, de Leeuw R. Prevalence of Suicidal Ideation, Depression, and Anxiety in Chronic Temporomandibular Disorder Patients. J Oral Facial Pain Headache. 2016;30(4):296-301.

https://doi.org/10.11607/ofph.1675

19. Brandini DA, Benson J, Nicholas MK, Murray GM, Peck CC. Chewing in temporomandibular disorder patients: an exploratory study of an association with some psychological variables. J Orofac Pain. 2011;25(1):56-67.

20. Yap AU, Chua E, Tan KB, Chan Y. Relationships between depression/somatization and self-reports of pain and disability. J Orofac Pain. 2004;18(3).

21. Slade G, Diatchenko L, Bhalang K, Sigurdsson A, Fillingim R, Belfer I, et al. Influence of psychological factors on risk of temporomandibular disorders. J Dent Res. 2007;86(11):1120-5. https://doi.org/10.1177/154405910708601119

22. Velly AM, Look JO, Carlson C, Lenton PA, Kang W, Holcroft $\mathrm{CA}$, et al. The effect of catastrophizing and depression on chronic pain--a prospective cohort study of temporomandibular muscle and joint pain disorders. Pain. 2011;152(10):2377-83.

https://doi.org/10.1016/j.pain.2011.07.004

23. Giannakopoulos NN, Keller L, Rammelsberg P, Kronmuller KT, Schmitter M. Anxiety and depression in patients with chronic temporomandibular pain and in controls. J Dent. 2010;38(5):369-76. https://doi.org/10.1016/j.jdent.2010.01.003

24. Reissmann DR, John MT, Seedorf H, Doering S, Schierz O. Temporomandibular disorder pain is related to the general disposition to be anxious. J Oral Facial Pain Headache. 2014;28(4):322-30.

25. Monteiro DR, Zuim PR, Pesqueira AA, Ribeiro Pdo P, Garcia AR. Relationship between anxiety and chronic orofacial pain of temporomandibular disorder in a group of university students. Journal of prosthodontic research. 2011;55(3):154-8.

https://doi.org/10.1016/j.jpor.2010.11.001

26. Tournavitis A, Tortopidis D, Fountoulakis K, Menexes G, Koidis P. Psychopathologic Profiles of TMD Patients with Different Pain Locations. Int J Prosthodont. 2017;30(3):251-7.

https://doi.org/10.11607/ijp.5155 